\title{
Per „Wissensmesse“ zum Forschungsprogramm - Arbeitsphasen und Abstimmungsprozesse zwischen Wissenschaft und Praxis
}

\author{
Regina Rhodius \& Michael Pregernig
}

\section{Der Kontext: Wofür das Verfahren gedacht und geeignet ist}

Mit dem Festlegen der Forschungsthemen und der Ausformulierung konkreter Forschungsfragen werden im Vorfeld und in den ersten Phasen eines Forschungsprojektes die Leitplanken für die folgende Bearbeitung gesetzt. Vor diesem Hintergrund haben insbesondere transdisziplinäre Forschungsprojekte den Anspruch, bereits die Forschungsthemen und -fragen in enger Zusammenarbeit von Wissenschaft und Praxis zu erarbeiten. Dies soll garantieren, dass die Forschungsprojekte sowohl zur Lösung praktischer Problemstellungen als auch zu neuen wissenschaftlichen Erkenntnissen beitragen (Lang et al. 2012; Moser 2016).

Üblicherweise wird in den Methodenkapiteln wissenschaftlicher Arbeiten zwar klar dargelegt, wie der Forschungsprozess selbst organisiert und von welchen Forschungsfragen dieser geleitet war. Demgegenüber bleibt aber oft im Dunkeln, woher die Initiative für ein Forschungsprojekt konkret stammte, wie das Team zusammenkam und wie Forschungsthemen und -fragen gefunden und ausformuliert wurden (Hoyningen-Huene 1987; Weingart 1993; Blättel-Mink et al. 2003). Gerade für das Verständnis und letztendlich auch die erfolgreiche Steuerung von inter- und transdisziplinären Projekten sind diese frühen Projektphasen jedoch von besonderer Bedeutung, weil sie den Rahmen der zukünftigen Zusammenarbeit von Wissenschaft und Praxis festlegen (Defila et al. 2006).

Mit diesem Beitrag - der Beschreibung der Vorbereitung, Durchführung und Nachbereitung der Wissensmesse - wollen wir daher ein Verfahren vorstellen, das der Erarbeitung eines transdisziplinären Forschungsprogramms (damit ist das Forschungsprogramm innerhalb eines Projekts oder Forschungsverbunds gemeint) im Austausch zwischen Wissenschaft und Praxis in diesen frühen Projektphasen dient. Dabei gehen wir prämissenhaft davon aus, dass der Definition von Forschungsfragen stets ein sozialer Aushandlungsprozess zugrundeliegt 
(Wiek 2007): Aufgrund ihrer spezifischen institutionellen Einbindung, ihrer unterschiedlichen Erfahrungen und persönlichen Motive haben sowohl Wissenschaftler(innen) als auch Praxisakteure jeweils unterschiedliche Erwartungen dazu, welche Forschungsthemen als relevant angesehen werden und wie sie bearbeitet werden sollten.

Vor dem Hintergrund der unterschiedlichen Interessen der beteiligten Akteure stellt sich die Frage, wie die Erarbeitung eines Forschungsprogramms so strukturiert werden kann, dass am Ende ein für Wissenschaft und Praxis gleichermaßen relevantes und von den beteiligten Akteuren als legitim empfundenes Forschungsprogramm vorliegt. Hierzu schlagen wir im Folgenden eine Abfolge von Arbeitsphasen und Abstimmungsprozessen vor, in deren Zentrum die Wissensmesse steht. Die vorgeschlagene Schrittfolge beinhaltet Elemente der Beteiligung im Sinne der Öffnung für Themenvorschläge sowie Elemente der Entscheidungsfindung im Sinne der Schließung des Themenfindungsprozesses. Das Vorgehen lehnt sich hinsichtlich Zielsetzung und Phasenfolge an das im Reallabor „Wissensdialog Nordschwarzwald" (WiNo, s. WiNo-Box 1, Steckbrief im Anhang zu diesem Buch und Bachinger et al. 2018) angewandte Vorgehen an ${ }^{1}$. Basierend auf den in WiNo gemachten Erfahrungen haben wir das Vorgehen zum Teil überarbeitet und angepasst. Unser Anliegen war es dabei, den in WiNo realisierten Ansatz der Wissensmesse zu einem praktikablen und dennoch durchgehend transdisziplinären Design weiterzuentwickeln. Während im Fließtext unser empfohlenes Vorgehen beschrieben wird, stellen wir in Textkästen beispielhaft dar, wie in WiNo konkret vorgegangen wurde.

Geeignet ist das beschriebene Vorgehen der Wissensmesse für Forschungskontexte, in denen sich Akteure aus Wissenschaft und Praxis unter dem Dach eines relativ breiten Oberthemas zusammenfinden, z. B. zu Fragen wie „Wie kann eine nachhaltige Energieversorgung eines Stadtquartiers aussehen?“ oder „Wie können die Mobilitätsoptionen im ländlichen Raum erhöht werden?“. Unter einem solchen Themendach lässt sich eine Vielzahl an spezifischeren Themen und Fragestellungen identifizieren. Dies macht ein systematisches Vorgehen notwendig, mit dessen Hilfe zentrale Themen herausgearbeitet, priorisiert und letztendlich in ein kohärentes Forschungsprogramm übersetzt werden. Die Wissensmesse eignet sich demgegenüber weniger für Kontexte, in denen - wie etwa im Fall klassischer

1 Die Autorin und der Autor des Beitrags waren im Rahmen der Arbeitsgruppe (s. WiNo-Box 2) sowie als WiNo-Geschäftsführerin bzw. Mitantragsteller von WiNo maßgeblich an der Vorbereitung, Durchführung und Nachbereitung der Wissensmesse beteiligt. Sie danken dem gesamten WiNo-Team unter Leitung von Frau Prof. Dr. Barbara Koch für die im Rahmen der WiNo-Wissensmesse ermöglichten Erfahrungen. Für wesentliche Impulse bei der Konzeptionierung der WiNo-Wissensmesse danken wir insbesondere den Beratern der EVOCO GmbH. 
Der Nationalpark Schwarzwald wurde im Januar 2014 gegründet. Seiner Ausweisung gingen kontroverse politische Debatten voran, die sich auf die ökologische Entwicklung des Gebietes und die Auswirkungen des Nationalparks auf die umliegende Region bezogen. Das Reallabor „Wissensdialog Nordschwarzwald“ (WiNo) wurde ein Jahr später mit dem Ziel ins Leben gerufen, den Nationalpark forschend zu begleiten und Impulse für die nachhaltige Entwicklung des Schutzgebietes und seiner Umgebung zu geben. Welche konkreten Themen und Forschungsfragen dabei im Vordergrund stehen würden, stand bei der Entscheidung der beiden beteiligten Hochschulen, die Finanzierung eines Reallabors beim baden-württembergischen Wissenschaftsministerium zu beantragen, noch nicht fest. Die Antragstellenden wollten diese in einem partizipativen Prozess gemeinsam mit Praxisakteuren und interessierten Kreisen der Zivilgesellschaft im Sinne einer breiten Öffentlichkeit erarbeiten.

Mit diesem schrittweisen Vorgehen sollten mehrere Ziele verfolgt werden: Der Prozess sollte (1) sicherstellen, dass in der Forschung die Erwartungen und Interessen der in der Region aktiven Praxisakteure sowie der dort lebenden Menschen so weit wie möglich berücksichtigt und diese (2) für eine aktive Zusammenarbeit mit der Wissenschaft gewonnen und mobilisiert werden. Gleichzeitig sollte (3) gewährleistet werden, dass Problemstellungen identifiziert werden, für deren Bewältigung Forschungsbeiträge tatsächlich notwendig sind (d. h. die nicht allein mit Beratung oder regionalen Förderinstrumenten zu, lösen' wären) und für welche die an der Antragstellung beteiligten Forschungsinstitutionen auch über die notwendige Expertise verfügen. Des Weiteren sollten (4) Anreize für die beteiligten Wissenschaftler(innen) geschaffen werden, im Reallabor konsequent transdisziplinär zu arbeiten. Zudem sollten (5) Forschungsergebnisse erzielt werden, die über die Projektregion Nordschwarzwald hinaus von Interesse und übertragbar auf andere Kontexte sind. Die Verfahrensschritte dieses Prozesses erstreckten sich in WiNo über 13 Monate, wobei ein Teil auf die (nicht finanzierte) Phase der Vorantragstellung, ein Teil auf die (anschubfinanzierte) Phase der Hauptantragstellung und mit neun Monaten der überwiegende Teil auf die erste Projektphase entfielen.

WiNo-Box 1: Das Anwendungsbeispiel „Wissensdialog Nordschwarzwald“ (WiNo) - Hintergrund und Zielstellung

Auftragsforschung - das konkrete Thema bereits weitgehend gesetzt ist und Forschungsfragen bereits klar eingegrenzt sind. Was den räumlichen Bezug betrifft, so lässt sich das Verfahren gleichermaßen in kleinräumigen Projektregionen, wie z. B. städtischen Quartieren, sowie in großflächigeren Räumen ländlicher Regionen oder in einem Stadt-(Um)Land-Kontext anwenden.

Das beschriebene Vorgehen bietet Methoden für die fokussierte Zusammenarbeit von Wissenschaftler(inne)n und Praxisakteuren. Mit Letzteren angesprochen ist dabei weniger eine ,breite Öffentlichkeit‘ im Sinne von vorab nicht näher mit dem Thema befasster Bürger(innen) (hier verstanden als die Bevölkerung; zu den Herausforderungen der Begrifflichkeit s. a. Defila und Di Giulio 2018b sowie Ar- 
nold und Piontek 2018). Die Wissensmesse zielt vielmehr einerseits auf eine ,organisierte Öffentlichkeit‘ im Sinne von Interessenvertretungen, Vertreter(inne)n zivilgesellschaftlicher Organisationen oder lokaler Einrichtungen und andererseits auf eine ,Fachöffentlichkeit' mit Vertreter(inne)n aus Verwaltung, Politik, Wirtschaft, Planung etc. (Arbter 2012). Der von uns vorgeschlagene, letztendlich sehr aufwendige und auf die transdisziplinäre Entwicklung eines Forschungsprogramms gerichtete Prozess scheint uns nur im Kreise einer solchen fachkundigen und organisierten Öffentlichkeit sinnvoll und realisierbar zu sein. Die breite Öffentlichkeit wird in einem derartigen Vorgehen eher punktuell und im Sinne des Informiert-Werdens eingebunden. Unter Praxisakteuren verstehen wir demzufolge Personen, die der organisierten Öffentlichkeit bzw. Fachöffentlichkeit angehören, damit im Themenfeld des Projektes über Praxis-Expertise verfügen und zu den Nachfragenden und Anwender(inne)n von Forschungsergebnissen gehören (s. a. Defila und Di Giulio 2018a). Als Wissenschaftler(innen) bzw. als zugehörig zur Wissenschaft bezeichnen wir in diesem Beitrag Personen, die an Universitäten, Hochschulen bzw. Forschungseinrichtungen im Bereich der Forschung tätig sind.

\section{Das Vorgehen: In 13 Schritten zum transdisziplinären Forschungsprogramm}

Die transdisziplinäre Erarbeitung eines Forschungsprogramms beginnt üblicherweise bereits mit der Vorbereitung eines Projektantrags und setzt sich bis in die ersten Projektmonate hinein fort. Das im Folgenden beschriebene Vorgehen umfasst 13 Schritte, die sich - wie in Tab. 1 dargestellt - in drei Phasen unterteilen lassen.

Für Phase I sind mindestens zwei Monate, für Phase II mindestens drei Monate und für Phase III mindestens zwei Monate zu veranschlagen. Damit benötigt das Vorgehen bei einer knappen zeitlichen Kalkulation insgesamt einen Zeitraum von mindestens sieben Monaten. Falls die zeitlichen und finanziellen Ressourcen es erlauben, empfehlen wir, für die Phase II, die Erarbeitung von Ideen für Teilprojekte, mehr Zeit - im Idealfall ein halbes Jahr - einzuplanen; der Gesamtprozess erstreckt sich in diesem Fall über mindestens zehn Monate. 
Tabelle 1: Phasenfolge des Vorgehens und beteiligte Akteure.

\begin{tabular}{|c|c|}
\hline Vorgehensschritt & Beteiligte Akteure \\
\hline \multicolumn{2}{|c|}{$\begin{array}{l}\text { Phase I: Etablierung der Projektstrukturen und Festlegung von Themenschwerpunk- } \\
\text { ten }\end{array}$} \\
\hline 1) Bilden eines Kernteams & Initiator(inn)en \\
\hline $\begin{array}{l}\text { 2) Befragen von Praxisakteuren zu ihren thema- } \\
\text { tischen Interessen }\end{array}$ & Kernteam \\
\hline 3) Festlegen von Themenschwerpunkten & Kernteam \\
\hline 4) Einsetzen eines Projektbeirats & Kernteam \\
\hline $\begin{array}{l}\text { 5) Erarbeitung von Auswahlkriterien für die } \\
\text { Teilprojekte }\end{array}$ & Kernteam, Projektbeirat \\
\hline 6) Ausschreiben von Themenschwerpunkten & Kernteam \\
\hline \multicolumn{2}{|c|}{$\begin{array}{l}\text { Phase II: Erarbeitung von Ideen für Teilprojekte und deren Präsentation auf der } \\
\text { Wissensmesse }\end{array}$} \\
\hline 7) Erarbeiten von Vorschlägen für Teilprojekte & Wissenschafts-Praxis-Teams \\
\hline 8) Vorauswahl geeigneter Ideen & Projektbeirat, Kernteam \\
\hline 9) Vorbereiten der Wissensmesse & $\begin{array}{l}\text { Kernteam, Wissenschafts-Praxis- } \\
\text { Teams }\end{array}$ \\
\hline 10) Durchführen der Wissensmesse & $\begin{array}{l}\text { Kernteam, Wissenschafts-Praxis- } \\
\text { Teams, Projektbeirat, Fachöffent- } \\
\text { lichkeit und organisierte Öffent- } \\
\text { lichkeit, z. T. breite Öffentlichkeit }\end{array}$ \\
\hline \multicolumn{2}{|c|}{$\begin{array}{l}\text { Phase III: Überarbeitung der Teilprojektvorschläge und Festlegung des Forschungs- } \\
\text { programms }\end{array}$} \\
\hline $\begin{array}{l}\text { 11) Übermitteln des Feedbacks des Projektbei- } \\
\text { rats und Ausarbeiten von Projektskizzen }\end{array}$ & $\begin{array}{l}\text { Projektbeirat, Wissenschafts- } \\
\text { Praxis-Teams }\end{array}$ \\
\hline $\begin{array}{l}\text { 12) Empfehlung des Projektbeirats und Festlegen } \\
\text { des Forschungsprogramms durch das Kern- } \\
\text { team }\end{array}$ & Projektbeirat, Kernteam \\
\hline $\begin{array}{l}\text { 13) Kommunikation des beschlossenen For- } \\
\text { schungsprogramms, Dokumentation und } \\
\text { Abschluss der Programmentwicklung }\end{array}$ & $\begin{array}{l}\text { Kernteam, Wissenschafts-Praxis- } \\
\text { Teams }\end{array}$ \\
\hline
\end{tabular}




\subsection{Phase I: Etablierung der Projektstrukturen und Festlegung von Themenschwerpunkten}

\section{Schritt 1: Bilden eines Kernteams}

Bereits in der Vorbereitungsphase (z. B. Antragstellung) empfehlen wir, ein paritätisch aus Wissenschaftler(inne)n sowie aus Praxisakteuren, d. h. Akteuren aus der Fachöffentlichkeit und der organisierten Öffentlichkeit, zusammengesetztes Kernteam zu bilden, das die Projektleitung enthält und später um die Geschäftsführung o. Ä. ergänzt wird (s. WiNo-Box 2). Dieses transdisziplinäre Kernteam bleibt über den gesamten Projektzeitraum bestehen und bildet das zentrale Steuerungsgremium des Projektes. Durch die paritätische Zusammensetzung sollte sichergestellt sein, dass sowohl wissenschaftliche als auch praxisbezogene Perspektiven und Kriterien ausreichend berücksichtigt werden.

Das Kernteam definiert das Dachthema des zukünftigen Projekts und sorgt für die Verankerung der Projektidee in den beteiligten Institutionen bzw. Gruppierungen.

In Reaktion auf die Ausschreibung eines Reallabor-Formats durch das baden-württembergische Wissenschaftsministerium bildete sich an der Fakultät für Umwelt und Natürliche Ressourcen der Albert-Ludwigs-Universität Freiburg eine kleine Arbeitsgruppe mit Mitgliedern aus zwei Kreisen: (1) Sozialwissenschaftler(inne)n mit Interesse an und Erfahrung im Design und der Durchführung transdisziplinärer Forschungsformate und (2) Forscher(inne)n mit Erfahrung in ,klassischer' angewandter Forschung in den Forstwissenschaften und im Feld des Landnutzungsmanagements. Diese erste Kerngruppe nahm frühzeitig Kontakt mit der Nationalparkverwaltung auf, um die Eignung des neu gegründeten Nationalparks als Fallstudie abzuklären und relevante Themenfelder auszuloten. Im Anschluss daran wurden weitere potentielle Partnerinstitutionen kontaktiert. Eine besondere Rolle fiel dabei der Hochschule für Forstwirtschaft Rottenburg (HFR) zu: Diese hatte in der ersten Ausschreibungsrunde ebenfalls einen Antrag auf Einrichtung eines Reallabors im Kontext des Nationalparks Schwarzwald eingereicht. Auf Empfehlung des Fördergebers wurden die beiden Konsortien im weiteren Verlauf der Antragstellung miteinander verbunden.

Das gesamte Konsortium bestand nach erfolgreich abgeschlossener Partner- und Themensondierung aus einem interdisziplinären Forschungsteam aus Angehörigen der Albert-Ludwigs-Universität Freiburg, der Hochschule für Forstwirtschaft Rottenburg, des Öko-Instituts e. V., der Forstlichen Versuchs- und Forschungsanstalt Baden-Württemberg und der EVOCO GmbH. Die Verwaltungen des Nationalparks Schwarzwald und des Naturparks Schwarzwald Mitte/Nord wirkten als Praxispartner mit. Alle Institutionen wurden mit Beginn des Projektes Mitglieder des zentralen WiNo-Steuerungsgremiums, das zusammen mit der an der Albert-Ludwigs-Universität Freiburg angesiedelten Projektleitung und Geschäftsführung die anfängliche Kerngruppe ersetzte.

WiNo-Box 2: Bildung des Kernteams und Zusammenstellung des Konsortiums in WiNo 
Die im Kernteam vertretenen zwei bis drei Wissenschaftler(innen) sollten sowohl inhaltlich im Dachthema verankert sein als auch Erfahrung im Bereich transdisziplinärer Forschung mitbringen. Als Vertreter(innen) aus der Fachöffentlichkeit bzw. der organisierten Öffentlichkeit sollten Personen ausgewählt werden, die gut im Praxisfeld vernetzt sind und deren Stimme dort Gewicht hat. Sollte sich das zukünftige Forschungsprojekt in einem stark konfliktbehafteten Themenfeld bewegen, empfiehlt es sich, im Kernteam Persönlichkeiten zu vereinen, die von den unterschiedlichen Konfliktparteien akzeptiert werden und eine hohe Integrationskraft mit sich bringen.

\section{Schritt 2: Befragen von Praxisakteuren zu ihren thematischen Interessen}

Das Kernteam initiiert eine Befragung von Praxisakteuren, um einen Überblick über die aus Sicht der Praxis relevanten Problemstellungen zu gewinnen. Am Anfang dieses Arbeitsschrittes steht eine Akteursanalyse, d. h. das systematische Identifizieren der am Dachthema interessierten Akteure. Methodisch eignen sich dafür z. B. Internetrecherchen, die Analyse von Dokumenten oder die Befragung von Personen, die als Schlüsselpersonen eingeschätzt werden. Zentral für die Auswahl der Akteure ist es, das gesamte Spektrum unterschiedlicher Meinungen und Interessen zum Dachthema abzubilden, um eine vorzeitige Festlegung auf Teilaspekte und vor allem thematische Schieflagen zu verhindern.

Aufbauend auf der Akteursanalyse werden die relevanten Akteure befragt (s. WiNo-Box 3). Die Anzahl der Befragten wird dabei je nach Größe des Bezugsraums und abhängig von der Breite und Diversität des Dachthemas schwanken. So kann es bei einem begrenzten Raum und einem klar umrissenen Thema ausreichen, fünf Akteure zu befragen, während größere Bezugsräume und ein fachlich noch wenig scharf gefasstes Thema die Befragung von zwanzig Akteuren erfordern können.

Je nach Thema, räumlichem Kontext und Ressourcenausstattung sind dafür sehr unterschiedliche methodische Zugänge, wie z. B. Expert(inn)eninterviews, Fragebögen oder die Durchführung von Expert(inn)enworkshops, vorstellbar. Bei der Auswahl der Methoden gilt es auch zu berücksichtigen, welche Wirkungen diese auf die befragten Akteure haben können. So bringt eine persönliche Befragung den Vorteil mit sich, das (angedachte) Forschungsformat im Raum, auf den sich das Projekt bezieht, bekannt zu machen und die Bereitschaft zum aktiven Mitgestalten zu wecken. Dabei muss aber darauf geachtet werden, dass keine überzogenen Erwartungen geweckt werden, die dann letztendlich nicht eingelöst werden können.

Die Methodenwahl ist auch mit der Frage der Finanzierung verbunden: Welche Mittel stehen - falls überhaupt - für eine Vorphase zur Verfügung, und wann 
Um die für die Region und deren nachhaltige Entwicklung tatsächlich relevanten Themen zu identifizieren, wurden in WiNo auf Initiative des Kernteams 19 regionale Praxisakteure in problemzentrierten Expert(inn)eninterviews nach den aus ihrer Sicht für die weitere nachhaltige Entwicklung der Region entscheidenden Themen befragt. Dabei wurden von den Befragten insgesamt 17 Themenfelder benannt. Daraus wurden auf Basis der Anzahl der Nennungen und der im Antragskonsortium vorhandenen Kompetenzen vom Kernteam folgende sechs Themenfelder für die weitere Bearbeitung ausgewählt:

1. Tourismus, nachhaltige Unternehmen und Markenbildung

2. Waldentwicklung und Naturschutz

3. Abiotische und biotische Störungen unter besonderer Berücksichtigung des Borkenkäfers

4. Kultur- und Naturgeschichte

5. Infrastruktur im Nationalpark

6. Soziale Vernetzung des Nationalparks in der Region (Querschnittsthema)

$\mathrm{Zu}$ jedem der sechs Themenfelder bildete sich eine Forschungsgruppe, die erste Forschungsfragen formulierte und diese später für die Präsentation auf der Wissensmesse weiterentwickelte. Anders als im jetzt vorgeschlagenen Verfahren wurden die Themenfelder in WiNo also nicht offiziell ausgeschrieben (s. Schritt 6), sondern von den am Thema interessierten Wissenschaftler(inne)n direkt bearbeitet.

WiNo-Box 3: Themensondierung in WiNo

steht fest, ob das Projekt (in der Hauptphase) gefördert wird oder nicht? Die Frage der für eine Vorphase zur Verfügung stehenden Mittel hat vor allem einen Einfluss darauf, wie umfassend und interaktiv die Befragung durchgeführt werden kann, und wie viel an Aktivierung der Akteure im Raum des Projekts damit vorab schon bewerkstelligt werden kann. Die Frage der Förderentscheidung hat einen Einfluss auf das ,Erwartungsmanagement': Je geringer die Wahrscheinlichkeit, dass das Projekt gefördert wird (z. T. auch, je später die Entscheidung über die Förderung fällt), desto vorsichtiger muss das Kernteam in der Befragung vorgehen, um keine falschen Erwartungen zu wecken.

\section{Schritt 3: Festlegen von Themenschwerpunkten}

Das Kernteam wertet die Ergebnisse der Befragung aus, bündelt Themenvorschläge und legt schließlich die thematischen Schwerpunkte innerhalb des Dachthemas fest. Dabei werden nicht nur die Priorisierung der Themen durch die Befragten berücksichtigt, sondern auch die folgenden Aspekte angemessen in die Auswahl einbezogen: 
- Eignung für transdisziplinäres Arbeiten: Bietet das Thema ausreichend Potential für transdisziplinäres Arbeiten? Ein Thema wird dann als in diesem Sinn potentialreich angesehen, wenn es sowohl wissenschaftliches Erkenntnisinteresse als auch praxisrelevante Fragestellungen adressiert und eine tatsächliche Zusammenarbeit von Wissenschaftler(inne)n und Praxisakteuren ermöglicht.

- Forschungsbedarf und -expertise: Handelt es sich um eine Herausforderung, $\mathrm{zu}$ deren Bewältigung Forschung auch tatsächlich einen relevanten Problemlösungsbeitrag leisten kann, oder erfordert es vielmehr andere Inputs (z. B. planerische Gutachten)? Lässt sich für das gewählte Thema die notwendige wissenschaftliche Expertise mobilisieren?

- Finanzierung: Wie viele Themenschwerpunkte lassen sich vor dem Hintergrund der (voraussichtlich) zur Verfügung stehenden Mittel in ausreichender Tiefe bearbeiten?

- Bandbreite der Themen: Erfordert das geplante Projekt eine besonders breite thematische Abdeckung (z. B. zur Analyse verschiedener Nachhaltigkeitsdimensionen) oder erscheint eine Fokussierung auf wenige zentrale Themen und damit ein schärferes Profil des Projektes sinnvoller? Lassen sich Themen zueinander in Beziehung setzen, so dass Themenbündel gebildet werden können?

Basierend auf der Erörterung dieser Fragen und Aspekte legt das Kernteam jene thematischen Schwerpunkte fest, die in den folgenden Schritten zur Ausschreibung gelangen sollen (s. Schritt 6).

\section{Schritt 4: Einsetzen eines Projektbeirats}

Aufbauend auf den Themenschwerpunkten stellt das Kernteam einen Projektbeirat aus externen Wissenschaftler(inne)n und Praxisakteuren zusammen. Die Größe des Gremiums sollte sieben bis neun Personen nicht überschreiten, um die Transaktionskosten für die Koordination des Gremiums überschaubar zu halten.

Als wissenschaftliche Mitglieder des Beirats ausgewählt werden sollten Vertreter(innen) wissenschaftlicher Disziplinen, die für die Bearbeitung des Dachthemas bzw. der Themenschwerpunkte als besonders wichtig angesehen werden, die selbst jedoch keine Forschungsabsichten im Projekt verfolgen. Dabei empfiehlt es sich, Forscher(innen) mit einem breiten, interdisziplinären Profil einzubeziehen, die bereits Erfahrung mit der Integration verschiedener Wissensbestände in ein übergeordnetes Thema mitbringen. Nach Möglichkeit sollten dem Projektbeirat zudem auch Wissenschaftler(innen) mit Erfahrungen in Transdisziplinarität angehören. Auch auf Seiten der Praxisakteure sollte auf eine ausreichende thematische Breite geachtet werden sowie darauf, dass keine Personen gewählt werden, die voraussichtlich in einem Teilprojekt mitwirken möchten. 
Der Projektbeirat ist ein Gremium mit Beratungsfunktion. Zentrale Aufgabe des Projektbeirats ist es, das Kernteam hinsichtlich der Auswahl von Teilprojekten zu beraten. Da der Beirat damit ein entscheidungsvorbereitendes Gremium ist, sollte bei der Auswahl seiner Mitglieder auf deren Neutralität bzw. Unbefangenheit bei Interessengegensätzen geachtet werden. Dies gilt umso mehr, je konfliktbehafteter das gewählte Dachthema ist.

\section{Schritt 5: Erarbeitung von Auswahlkriterien für die Teilprojekte}

Das vorgeschlagene Vorgehen sieht im Kern ein kompetitives Element bei der Auswahl von Teilprojekten vor. Der Wettbewerbsansatz soll den beteiligten Wissenschaftler(inne)n und Praxisakteuren einen Anreiz bieten, ihre Forschungsideen zu transdisziplinären Forschungsprojekten zu entwickeln. Für die Auswahl bzw. Priorisierung von Themenschwerpunkten und Teilprojekten braucht es klare Kriterien, die in einem transparenten Prozess erarbeitet und frühzeitig, d. h. zusammen mit der Ausschreibung von Themenschwerpunkten, kommuniziert werden. In prozeduraler Hinsicht schlagen wir vor, dass das Kernteam einen Vorschlag für ein Set an Kriterien erarbeitet, dieses Set dem Projektbeirat zur Kommentierung und Ergänzung vorlegt und abschließend über das finale Kriterienset entscheidet.

Inhaltlich sollten die Kriterien mehrere Zieldimensionen abdecken: Sie sollten sowohl die Praxisrelevanz als auch den wissenschaftlichen Mehrwert der anvisierten Teilprojekte adressieren und deren transdisziplinären Anspruch abbilden. Die in WiNo zur Anwendung gebrachten drei Kriterienkategorien haben sich in dieser Hinsicht sehr gut bewährt. Für die Entscheidungsfindung im Projektbeirat empfiehlt es sich, die Kriterien mit einer Skala (z. B. von eins bis fünf) zu versehen und die Kriterien zu gewichten (s. WiNo-Box 4).

\section{Schritt 6: Ausschreibung von Themenschwerpunkten}

Die in Schritt 3 festgelegten Themenschwerpunkte kommen nun zur Ausschreibung. Dazu formuliert das Kernteam einen Ausschreibungstext, der einerseits die zur Auswahl stehenden Themen spezifiziert und andererseits die in Schritt 5 erarbeiteten Auswahlkriterien festschreibt. Die Ausschreibung richtet sich explizit an Wissenschafts-Praxis-Teams und wird gezielt über Aushänge, Anschreiben, EMail-Verteiler u. Ä. in den relevanten Praxis- und Wissenschaftsinstitutionen beworben. Das Kernteam bietet Wissenschaftler(inne)n und Praxisakteuren zudem Unterstützung bei der Suche nach geeigneten Teampartner(inne)n an. 
In WiNo wurden drei zentrale Kriterien für die Priorisierung und Auswahl von Teilprojekten zur Anwendung gebracht. Die Kriterien wurden vom Kernteam erarbeitet und in ein Formular übersetzt. Dieses Formular diente den Teilprojektgruppen zur Einreichung ihrer Teilprojektskizzen im Nachgang der Wissensmesse. Ziel war, mit diesen drei Kriterien die wesentlichen Reallabor-Prinzipien Co-Design und Co-Produktion (für Weiteres zu den Merkmalen von Reallaboren s. Arnold und Piontek 2018 sowie Beecroft et al. 2018) abzubilden:

1. Gesellschaftliche Relevanz des Themenfeldes bzw. der Forschungsfragen:

Ist das jeweilige Themenfeld bzw. sind spezifische Forschungsfragen relevant für die Akteure vor Ort? Sind sie relevant für die zukünftige nachhaltige Entwicklung der Region Nordschwarzwald, d. h. haben sie das Potential, zur intendierten Nachhaltigkeitstransformation beizutragen?

2. Wissenschaftliche Umsetzbarkeit und Innovation/wissenschaftlicher Mehrwert:

Sind die Fragen einer wissenschaftlichen Bearbeitung (z. B. im Rahmen eines Dissertationsprojekts oder Forschungsauftrags) zugänglich und in der gegebenen Zeit umsetzbar? Sind die Themen und Fragen auch aus wissenschaftlicher Perspektive heraus interessant bzw. innovativ? Versprechen die Fragen einen wissenschaftlichen Mehrwert (gehen also z. B. über klassische Beratungsaufträge oder Datenerhebungen hinaus)?

3. Potential für eine transdisziplinäre Bearbeitung des Themenfeldes:

Erlaubt die Art der Konzeption des Themenfeldes bzw. der Forschungsfrage die zukünftige aktive Einbeziehung der Akteure vor Ort in die Forschungsarbeiten? ,Aktiv' meint hier, dass regionale Akteure und Bürger(innen) nicht nur befragt bzw. Organisationen nicht nur untersucht werden, sondern dass sie zu aktiven Co-Produzent(inn)en von Wissen werden.

Der Projektbeirat erhielt einen auf diesen Kriterien aufbauenden Bewertungsbogen und bewertete die Erfüllung der drei Kriterien auf einer Skala von eins (= außerordentlich gut erfüllt) bis fünf (= nicht erfüllt). Zudem gewichtete er die Kriterien für jeden Teilprojektvorschlag, d. h. er konnte z. B. die gesellschaftliche Relevanz eines Vorschlags über den wissenschaftlichen Mehrwert stellen oder umgekehrt. Abschließend diskutierten die drei Beiratsmitglieder ihre Bewertung und einigten sich auf eine gemeinsame Priorisierung der Vorschläge. Diese Priorisierung folgte nicht durchgängig den Gesamtpunktzahlen, die die Vorschläge durch das Addieren der von jedem Mitglied vergebenen Punkte erhalten hatten, sondern entstand teilweise auch als Ergebnis eines argumentativen Aushandlungsprozesses zwischen den Beiratsmitgliedern.

WiNo-Box 4: Kriterien zur Beurteilung der Skizzen für WiNo-Teilprojekte 


\subsection{Phase II: Erarbeitung von Ideen für Teilprojekte und deren Präsentation auf der Wissensmesse}

\section{Schritt 7: Erarbeiten von Vorschlägen für Teilprojekte}

Auf der Basis der ausgeschriebenen Themenschwerpunkte und Kriterien entwickeln die Teams aus Wissenschaftler(inne)n und Praxisakteuren ihre Ideen für Teilprojekte. Als Teamleitung fungieren können dabei gleichermaßen Akteure aus Praxis oder Wissenschaft.

Die von den Teams skizzierten Vorschläge bilden die Grundlage für die Auswahl der Teilprojekte durch Projektbeirat und Kernteam. Die Teilprojektvorschläge stehen untereinander im Wettbewerb um die Finanzierung. Um den Wettbewerb nicht auf Alles-oder-Nichts-Entscheidungen zu reduzieren, sollte den Wissenschafts-Praxis-Teams empfohlen werden, in den Teilprojektvorschlägen Forschungspakete zu erarbeiten, die das Bewilligen kleinerer oder größerer Forschungsvorhaben ermöglichen.

\section{Schritt 8: Vorauswahl geeigneter Ideen}

Der Projektbeirat beurteilt die eingereichten Vorschläge auf Basis der in Schritt 5 entwickelten Kriterien und empfiehlt dem Kernteam Teilprojektideen für die Vorauswahl. Das Kernteam entscheidet und lädt die ausgewählten sechs bis acht Teams zur Weiterentwicklung ihrer Teilprojekt-Ideen und deren Präsentation in der Wissensmesse ein. Mit der Auswahl der Ideen verbunden ist die Bewilligung einer Aufwandsentschädigung für Personal- und Sachkosten, die von den Wissenschafts-Praxis-Teams zur Vorbereitung der Präsentation in der Wissensmesse und ggf. zur anschließenden Weiterentwicklung ihrer Vorschläge benötigt werden.

\section{Schritt 9: $\quad$ Vorbereitung der Wissensmesse}

Die Wissensmesse bildet das ,Herzstück' der vorgeschlagenen Schrittfolge. Hier wird die breit gefächerte Expertise der Fachöffentlichkeit sowie der organisierten Öffentlichkeit in die Erstellung des Forschungsprogramms integriert; hier können Praxisakteure, die nicht an der Ausarbeitung von Teilprojekt-Ideen beteiligt waren, entscheidenden Einfluss auf die Auswahl und weitere Konkretisierung von Themenschwerpunkten nehmen. Zugleich ,betritt' das Projekt mit der Wissensmesse ,auf breiter Front' den öffentlichen Raum, will neugierig machen auf die zukünftige Forschung und zur Mitarbeit einladen. Diese Ziele prägen auch die Vorbereitung der Wissensmesse, die in den Händen des Kernteams liegt. Die Vorbereitung beinhaltet sowohl a) die Auswahl und Einladung von Akteuren, b) die inhaltliche Konzeption und c) die praktische Vorbereitung der Veranstaltung. 
a) Auswahl und Einladung von Akteuren: Anspruch der Wissensmesse ist es, über die bereits in den Wissenschafts-Praxis-Teams involvierten Praxisakteure hinaus die im Projektraum vorhandene Fachöffentlichkeit und organisierte Öffentlichkeit zu erreichen und deren Expertise zum Dachthema sowie zu den ausgewählten Themenschwerpunkten zu bündeln. Die in Schritt 8 ausgewählten Teams werden daher gebeten, dem Kernteam Akteure zu benennen, die aus ihrer Sicht geeignete Gesprächspartner(innen) für die Weiterentwicklung des von ihnen vorgeschlagenen Teilprojektes sind. Zugleich führt das Kernteam eine Akteursanalyse durch, die auf der in Schritt 2 bereits stattgefundenen Analyse aufbaut. Die ausgewählten Akteurskreise werden persönlich eingeladen (per E-Mail, Anschreiben); zugleich werden Multiplikator(inn)en angesprochen, die zu einem hohen Bekanntheitsgrad der Wissensmesse beitragen können. Es empfiehlt sich, dass die Einladungen sowohl zentral vom Gesamtprojekt als auch mit direktem thematischem Bezug von den ausgewählten Wissenschafts-Praxis-Teams ausgesprochen werden. Interessierte Bürgerinnen und Bürger im Sinne der breiten Öffentlichkeit wiederum werden durch eine dem Kontext entsprechende Presse- und Öffentlichkeitsarbeit angesprochen und eingeladen (z. B. über lokale Medien, Aushänge und Flyer in Läden, Banken, Gemeindeverwaltungen, Schulen etc.).

Die Wissensmesse erhält ihre inspirierende Atmosphäre sowie ihre Wirkkraft erst bei einer ausreichenden Anzahl an Teilnehmenden. Die konkrete Anzahl macht sich an der Anzahl der Themenschwerpunkte und der Größe des Projektraumes fest, sollte jedoch 50 externe Personen, d. h. solche, die nicht zu den eingeladenen Wissenschafts-Praxis-Teams, zum Kernteam und zum Projektbeirat gehören, nicht unterschreiten. Deshalb empfiehlt es sich, ausreichend Zeit für die Mobilisierung von Teilnehmenden einzuplanen, um ggf. mehr als eine Einladungsrunde durchführen zu können.

b) Inhaltliche Konzeption: Das Programm der Wissensmesse muss verschiedenen Ansprüchen gerecht werden: Es soll zum einen attraktiv und abwechslungsreich sein, um die Teilnehmenden neugierig auf die Veranstaltung zu machen, zur Mitarbeit zu motivieren und eine gute Arbeitsatmosphäre zu schaffen. Zum anderen muss es geeignete Methoden vereinen, um über das Projekt zu informieren, Raum für die Vorstellung und Diskussion der Teilprojekt-Ideen der Wissenschafts-Praxis-Teams zu geben und die Prioritäten der Teilnehmenden in Bezug auf die zukünftigen Forschungsfragen zu erfahren (s. Schritt 10). Die Auswahl dieser Methoden und deren Moderation während der Veranstaltung erfordern Kompetenzen und Erfahrung in der Arbeit mit Großgruppen. Zur Arbeit mit Großgruppen existiert ein breites Spektrum an Literatur (z. B. Will et al. 2009; Königswieser und Keil 2000; Seliger 2008). Hier ist die Entscheidung des Kernteams gefragt, ob es selbst über diese Kompetenzen verfügt oder die Beauftragung von Kommunikationsexpert(inn)en erforderlich ist. Um ausreichend Zeit für 
Die Wissensmesse wurde von der zu diesem Zeitpunkt bereits eingesetzten WiNo-Geschäftsführung in Zusammenarbeit mit einer Arbeitsgruppe, die Vertreter(innen) aller Themenfelder enthielt, und dem für die Moderation der Veranstaltung verantwortlichen WiNo-Partner EVOCO GmbH in einem Zeitraum von knapp fünf Monaten vorbereitet.

Innerhalb dieser Zeit erarbeiteten die Themenfeldteams ihre Projektskizzen; teilweise fanden auch bereits erste bilaterale Abstimmungen mit der Nationalparkverwaltung und weiteren Praxisakteuren statt. Das Format der eintägigen Veranstaltung, der Kreis der einzuladenden Akteure und die Art der Präsentation und Diskussion der TeilprojektIdeen wurden zwischen allen Beteiligten abgestimmt. Die Arbeitsgruppe erwies sich dabei als geeignetes Format für den themenfeldübergreifenden Austausch und wurde im weiteren Projektverlauf als Querschnittsarbeitsgruppe fortgeführt. Im Vorfeld der Wissensmesse führte sie fünf Arbeitstreffen durch (Dauer jeweils ca. 2,5 h).

Wenige Tage vor der Veranstaltung fand ein ,Probelauf' statt, bei dem der Verlauf der Veranstaltung noch einmal durchgesprochen wurde und die beteiligten Forschenden kollegiales Feedback in Bezug auf die von innen vorbereitete Präsentation ihrer Teilprojekt-Ideen (Vortrag und Poster) erhielten. Mittels lokaler und regionaler Medien sowie Aushängen in den Gemeinden der Region Nordschwarzwald wurde die Wissensmesse beworben.

WiNo-Box 5: Ausarbeitung der Forschungsideen und Vorbereitung der Wissensmesse

die verschiedenen Facetten der Veranstaltung zu haben, sollte mindestens ein halber Tag (d .h. vier Stunden) veranschlagt werden. Die Höchstdauer sollte sich am Zeitrahmen orientieren, den sich Akteure aus Wissenschaft und Praxis für eine solche Veranstaltung nehmen (können), und deshalb in der Regel nicht über einen Tag hinausgehen.

Bei der Programmerstellung ist es auch wichtig, die Rückkopplung mit den Wissenschafts-Praxis-Teams zu suchen, da diese hinter der Wissensmesse und den eingesetzten Methoden stehen sollten, um die Veranstaltung mit Leben füllen zu können. Die Kerngruppe organisiert vorbereitende Treffen der WissenschaftsPraxis-Teams, bei denen Ziele, Ablauf und Art der Präsentation der TeilprojektIdeen durchgesprochen werden (s. WiNo-Box 5). Eine gründliche Vorbereitung ist insbesondere deshalb wichtig, weil die Art der Präsentation stark von den auf wissenschaftlichen Konferenzen üblichen Präsentationsformen abweicht (s. Schritt 10).

c) Praktische Vorbereitung: Die Vorbereitung der Wissensmesse beinhaltet die Organisation geeigneter Räumlichkeiten. Der Veranstaltungsort sollte so gewählt werden, dass er - im Fall eines konfliktbehafteten Projektkontextes - politisch nicht vorbelastet, für den anvisierten Teilnehmendenkreis gut erreichbar ist und einen Wechsel zwischen der Arbeit in Kleingruppen und dem Zusammenkommen 
im Plenum zulässt. Die praktische Vorbereitung umfasst zudem die Organisation der an die gewählten Arbeitsmethoden angepassten technischen Infrastruktur, Catering und ggf. die Organisation von Kinderbetreuung, um auch Erziehenden die Teilnahme zu ermöglichen.

\section{Schritt 10: Durchführung der Wissensmesse}

Wie in Schritt 9 bereits geschildert, dient die Wissensmesse verschiedenen Zielen. Diese Vielfalt spiegelt sich auch in ihrem Ablauf wider, der die folgenden Programmpunkte beinhaltet:

\section{Ankunft und Willkommen}

Bereits eine halbe Stunde vor der offiziellen Begrüßung können die ersten Teilnehmenden eintreffen und bei Getränken und Snacks ins Gespräch kommen und sich umschauen. Zu diesem Zeitpunkt sind die Wissenschafts-Praxis-Teams, das Kernteam und der Projektbeirat bereits vor Ort und gesprächsbereit. Plenumssaal, Workshop-Räume und Marktplatz sind vollständig eingerichtet (Erläuterung zum Marktplatz s. u.).

\section{Begrüßung}

Die Begrüßung wird durch eine(n) Vertreter(in) des Kernteams oder eine(n) externe(n) Moderator(in) übernommen. Es empfiehlt sich, in die Begrüßung ein aktivierendes Element zu integrieren (z. B. mit der Bitte um Handzeichen oder Aufstehen, wenn man sich angesprochen fühlt bei Fragen wie „Wer von Ihnen ist weiter als XY km angereist?“; , Wer von Ihnen hat in seinem Alltag mit Thema $\mathrm{XY}$ zu tun?"). Die Teilnehmenden erhalten so einen ersten Überblick über den Kreis der Anwesenden und fühlen sich in gewisser Weise persönlich angesprochen. Zudem bietet die Abfrage im Sinne eines, Warm-up“ die erste Möglichkeit einer Interaktion, die zugleich auf die erwünsche Atmosphäre von Offenheit und Dialogbereitschaft hinwirken kann. Anschließend folgt ggf. die Begrüßung durch eine(n) Vertreter(in) des gastgebenden Ortes.

\section{Einführung in den Veranstaltungsablauf und das Projekt}

Der bzw. die Moderator(in) gibt einen Überblick über den Verlauf der Veranstaltung. Ein(e) Vertreter(in) des Kernteams stellt das Gesamtprojekt vor. Hier ist darauf zu achten, dass diese Einführung so kurz wie möglich (max. 10 Minuten) und auch möglichst anschaulich gehalten wird.

\section{Statements wichtiger Repräsentant(inn)en}

Je nach Thema und Umfang des Gesamtprojektes kann es sinnvoll sein, an dieser Stelle wichtige Repräsentant(inn)en aus Wissenschaft und Praxis zu Wort kommen zu lassen. Dies dient sowohl dazu, der Veranstaltung ein besonderes Gewicht zu verleihen, als auch dazu, das ,Commitment' zentraler Akteure für das 
Projekt zu fördern. Um eine ermüdende und für eine interaktiv ausgerichtete Veranstaltung eher kontraproduktive Aneinanderreihung von Grußworten zu vermeiden, empfiehlt sich die Form von Interviews, bei dem die Moderation gezielte Fragen an die max. drei Repräsentant(inn)en richtet. Je nach zeitlichem Rahmen der Veranstaltung kann hier dem Plenum die Möglichkeit für Rückfragen geboten werden.

\section{Ggf. Improvisationstheater vorsehen}

Um die Atmosphäre aufzulockern und die Erwartungen der Teilnehmenden wie auch die besprochenen Themen und die Art, wie diskutiert wird, zu spiegeln, kann die Einbindung eines Improvisationstheaters sinnvoll sein (s. Abb. 1 und WiNo-Box 6).

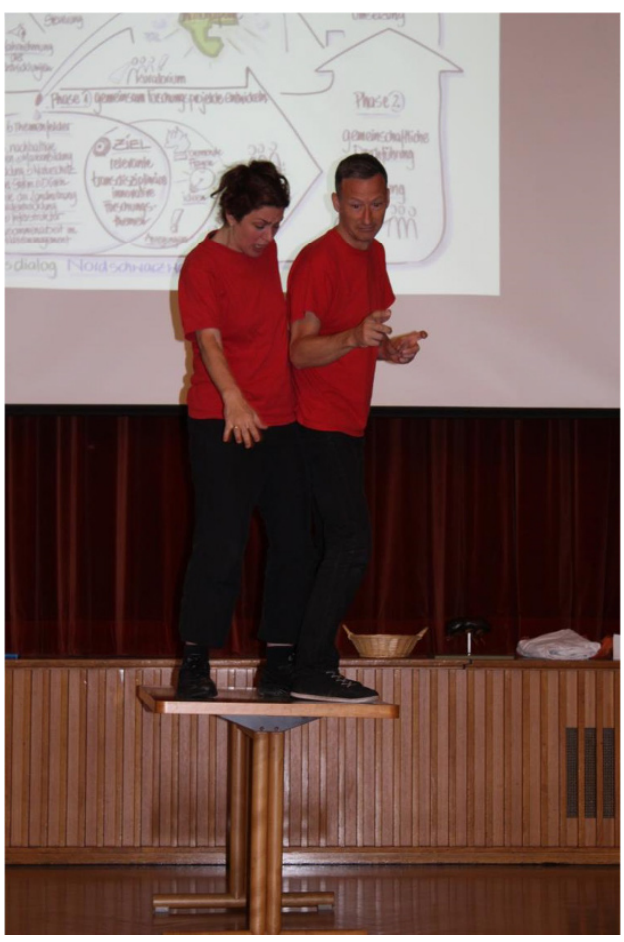

Abbildung 1: Schauspieler(innen) der Theatergruppe FREISTIL aus Freiburg i. B. banden auf der Wissensmesse von WiNo die Erwartungen der Teilnehmenden an die Veranstaltung in ihre Sketche ein. (C) WiNo 2015. 
Die Wissensmesse fand am 20. Juni 2015 unter dem Motto „Wissensdialog Nordschwarzwald - fragen, diskutieren, forschen" im K(ult)urhaus Bad Peterstal statt. Über 70 Personen aus der Region - vorwiegend Vertreter(innen) regionaler Institutionen und Interessengruppen - folgten der Einladung. Die Veranstaltung bot die im Text beschriebene Mischung aus Plenums- und Arbeitsgruppenphasen. Als sehr hilfreich mit Blick auf die Atmosphäre erwies sich die Einbindung eines Improvisationstheater-Duos (s. Abb. 1). Bereits vor Beginn der Veranstaltung ließen sich diese von den Teilnehmenden deren Erwartungen an die Veranstaltung in einem Satz auf Zettel notieren. Diese Sätze sowie Hintergrundinformationen, die sie im Vorfeld der Veranstaltung vom Kernteam erhalten hatten, und während der Begrüßung gewonnene Eindrücke flossen in die Darbietung des Schauspieler-Duos ein. So gelang es, auf heitere und leichte Art und Weise bestehende Ressentiments ,auf's Korn zu nehmen'. Die Anwesenden fanden sich in den dargestellten Szenen wieder. Gleichzeitig verband das Lachen auch gegensätzliche ,Meinungslager', die sich in der dem Projekt vorangegangenen Debatte um die Gründung des Nationalparks Schwarzwald herausgebildet hatten und den Projektstart prägten.

WiNo-Box 6: Improvisationstheater auf der Wissensmesse

\section{Vorstellung des weiteren Ablaufs}

Die Moderation stellt die nun folgenden Programmpunkte vor. Sie erläutert zugleich, welche Funktion diese haben, wie gearbeitet wird und wie die Ergebnisse der gemeinsamen Arbeit verwendet werden.

7. Kurzpräsentation der Teilprojektvorschläge durch die Wissenschafts-PraxisTeams

Da die Teilnehmenden entscheiden können, in welchen thematischen Workshops sie sich einbringen wollen, bedarf es vorab einer Kurzvorstellung der einzelnen Teilprojektvorschläge. Dazu werben die Wissenschafts-Praxis-Teams in einer kurzen Rede für die Teilnahme an ihren Workshops. Die Redezeit ist streng reglementiert (zwei bis max. fünf Minuten). Jedes Team visualisiert sein Thema innerhalb einer standardisierten Präsentation mit Hilfe eines Fotos bzw. einer Abbildung.

8. Vorstellung und Diskussion der Teilprojektvorschläge in zwei aufeinanderfolgenden Workshops

Die Wissenschafts-Praxis-Teams präsentieren und diskutieren ihre Teilprojektvorschläge in zwei aufeinanderfolgenden Workshops (ein Vorschlag pro Workshop, jeder Vorschlag wird also zweimal zur Diskussion angeboten). Die Diskussion wird von zwei Fragen geleitet, die den Teilnehmenden von den Wissenschafts-Praxis-Teams gestellt werden: 1) „Welchen Fragen sollten wir im Rahmen unserer Forschung vordringlich nachgehen?“ und 2) „Welche konkreten Anregungen, Hinweise und Empfehlungen wollen Sie uns mitgeben?“. Die Er- 
gebnisse der Diskussion in Form von Forschungsfragen und Anregungen werden auf vorbereiteten Blanko-,Sprechblasen' notiert und auf Ergebnis-Poster geklebt. In der zweiten Workshop-Phase können sich die Teilnehmenden für einen anderen Workshop entscheiden oder das gleiche Thema noch einmal besuchen.

\section{Marktplatzbesuch}

In der Pause zwischen den beiden Workshops (Mittagspause bei Ganztagsveranstaltung oder lange Kaffeepause bei Halbtagsveranstaltung) können die Teilnehmenden den Marktplatz besuchen. Als Marktplatz wird der Raum bezeichnet, in dem die Wissenschafts-Praxis-Teams ihre Teilprojekt-Ideen anhand von jeweils zwei Posterwänden präsentieren. Die Darstellung der Ideen auf den Postern soll sich auf eine knappe und anschaulich bebilderte Schilderung von Ausgangslage, Forschungsanlass und Ideen für Forschungsfragen beschränken. Auf einer dritten Posterwand können die Teilnehmenden ihre Anregungen, Fragen und Kommentare bereits vor Beginn der Veranstaltung, insbesondere aber bei dem nun stattfindenden Marktplatzbesuch notieren.

\section{Vorstellung der Workshop-Ergebnisse und Priorisierung von Forschungs- fragen}

Im Anschluss an den zweiten Workshop und eine kurze Pause, in der der Marktplatz nochmals besucht werden kann, findet die Präsentation der Workshop-Ergebnisse durch eine(n) Vertreter(in) des jeweiligen Wissenschafts-Praxis-Teams, ggf. auch durch eine(n) Teilnehmer(in) der einzelnen Workshops statt. Tauchen bei der Ergebnispräsentation noch neue, zuvor nicht genannte Forschungsfragen auf, so werden diese zusätzlich aufgenommen. Nach jeder Ergebnispräsentation schließt sich eine kurze Möglichkeit für Ergänzungen, Rückfragen oder Kommentare an.

Abschließend erhalten die Teilnehmenden die Gelegenheit, die Forschungsfragen zu priorisieren (s. Abb. 2 und 3). Hierzu erhält jede(r) Teilnehmer(in) verschiedenfarbige Klebepunkte, pro vorgeschlagenes Teilprojekt eine Farbe. Damit erhalten die Teilnehmenden die Möglichkeit, zu jedem Thema die aus ihrer individuellen Sicht wichtigste Forschungsfrage zu kennzeichnen. Wer bezogen auf ein Thema keinen Forschungsbedarf sieht (oder die Forschungsfragen für sich als nicht relevant oder treffend einschätzt), verzichtet in diesen Fällen auf das Punkten.

Ggf. erhalten die Teilnehmenden zusätzlich einen ,Jokerpunkt` zur Kennzeichnung der über alle Themen hinweg aus ihrer individuellen Sicht wichtigsten Forschungsfrage. 


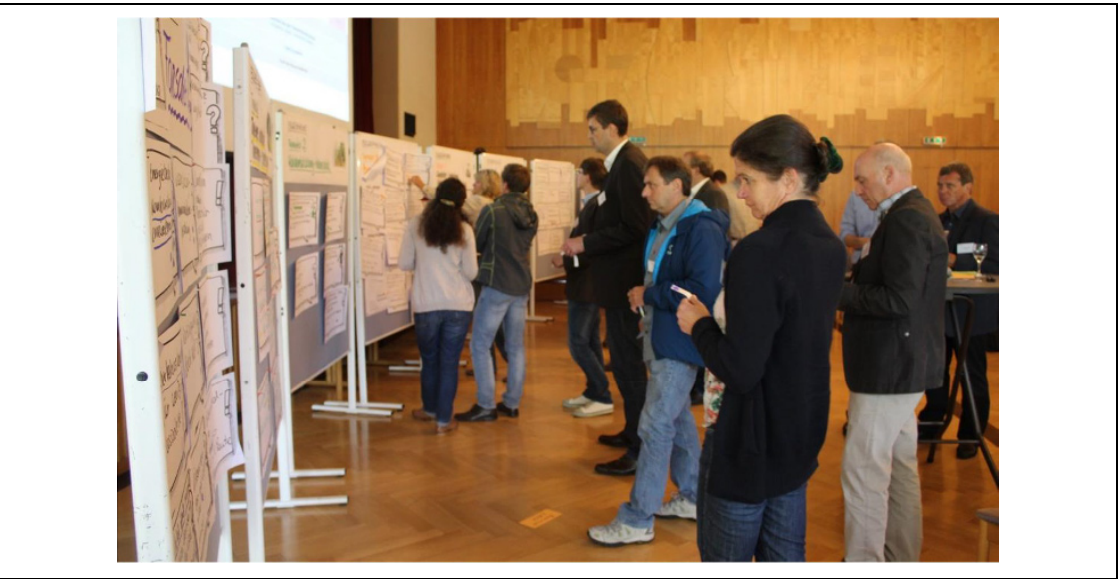

Abbildung 2: Im Anschluss an die Vorstellung der Workshop-Ergebnisse priorisierten die Teilnehmenden der WiNo-Wissensmesse die Forschungsfragen. (C) WiNo 2015.

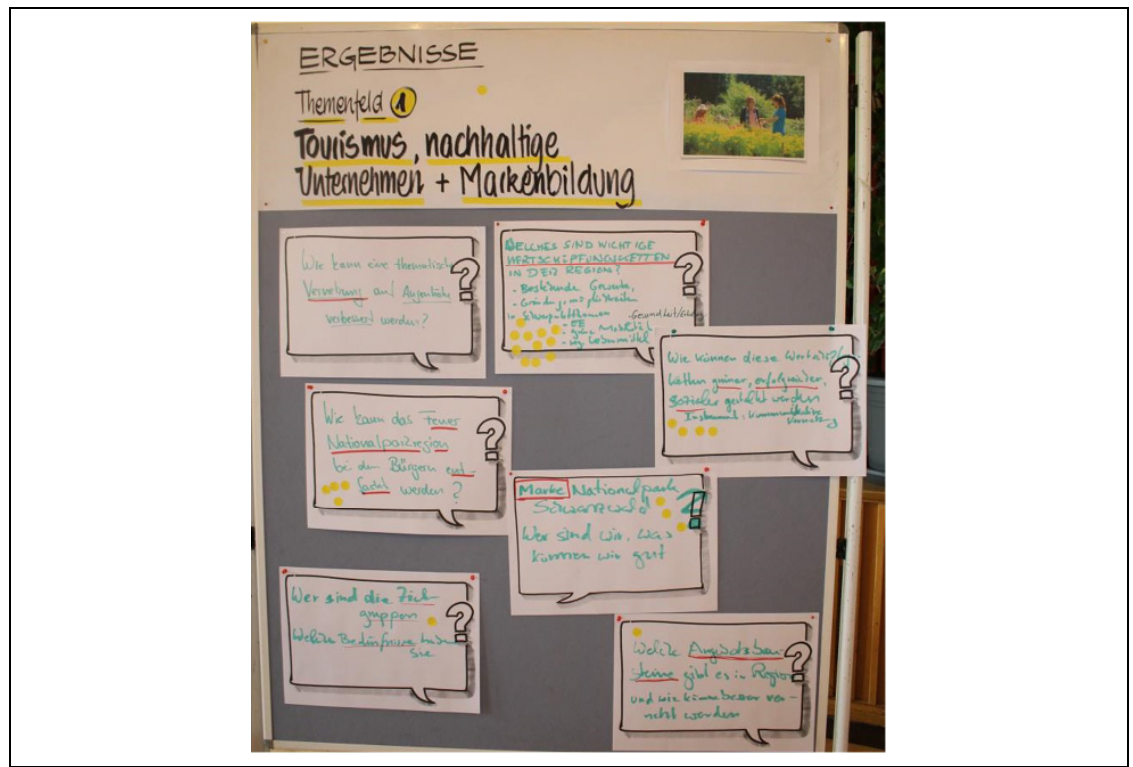

Abbildung 3: Die Anzahl der gelben Punkte auf den ,Sprechblasen“ macht deutlich, welche Fragen den Teilnehmenden der WiNo-Wissensmesse im Themenfeld Tourismus, nachhaltige Unternehmen und Markenbildung besonders wichtig waren. (C) WiNo 2015. 


\section{Fazit, Ausblick und Ausklang}

Zum Schluss gibt ein(e) Vertreter(in) des Kernteams einen Ausblick auf den Umgang mit den Ergebnissen, kündigt an, wann die Entscheidung über die Bewilligung der Teilprojekte fällt und wie sie bekanntgegeben wird, und bedankt sich bei den Teilnehmenden. Die Moderation knüpft daran an und holt Stimmen der Teilnehmenden ein zu Verlauf und Ergebnis der Wissensmesse und zu Hinweisen für die zukünftige Projektarbeit. Gestützt darauf kann an dieser Stelle evtl. ein letztes Mal eine Improvisationstheater-Gruppe zum Einsatz kommen. Die Teilnehmenden sowie die Wissenschafts-Praxis-Teams werden abschließend zu einem lockeren Ausklang bei Snacks und Getränken eingeladen.

\subsection{Phase III: Überarbeitung der Teilprojektvorschläge und Festlegung des Forschungsprogramms}

Schritt 11: Übermitteln des Feedbacks des Projektbeirats und Ausarbeitung von Projektskizzen

Im Anschluss an die Wissensmesse übermitteln die Mitglieder des Projektbeirates den Wissenschafts-Praxis-Teams sowie dem Kernteam ihr auf den Veranstaltungseindrücken basierendes Feedback sowie Hinweise zur Weiterentwicklung der einzelnen Teilprojekt-Ideen. Danach haben die Wissenschafts-Praxis-Teams bis zu max. vier Wochen Zeit, um ihre Teilprojektvorschläge anhand eines Fragenkataloges (s. WiNo-Box 7) zu überarbeiten. Dieser Fragenkatalog basiert auf den in Schritt 5 ausgearbeiteten Kriterien, zielt aber vor allem auf die Einbindung der Ergebnisse der Wissensmesse in die Teilprojektvorschläge der WissenschaftsPraxis-Teams. Der Umfang der Projektskizzen umfasst fünf bis maximal zehn Seiten. Parallel dazu werden die Ergebnisse der Wissensmesse mit Hilfe von Fotos und/oder Protokollnotizen belegt. Das Kernteam steht den WissenschaftsPraxis-Teams bei der Erstellung der Skizzen beratend zur Seite. Die Skizzen werden über das Kernteam dem Projektbeirat eingereicht.

\section{Schritt 12: Empfehlung des Projektbeirats und Festlegen des Forschungsprogramms durch das Kernteam}

Der Projektbeirat bewertet die Projektskizzen anhand der in Schritt 5 erarbeiteten Kriterien und unterbreitet dem Kernteam einen Vorschlag, welche Teilprojektvorschläge gefördert werden und welche dabei bei der Budgetverteilung prioritär behandelt werden sollten. Darüber hinaus kann der Projektbeirat zuhanden der von ihm priorisierten Teilprojekte Empfehlungen für die Forschungsarbeit übermitteln. 
Auf der Basis dieser Empfehlungen legt das Kernteam das Forschungsprogramm und die darin enthaltenen einzelnen Teilprojekte fest. Es vergibt dabei ggf. auch gesonderte Mittel für Instrumente, die speziell transdisziplinären Arbeitsweisen dienen sollen (z. B. Ressourcen für die Zusammenarbeit mit Akteuren in Form von Workshops, gemeinsamen Wissenschafts-/Praxis-Veranstaltungen oder der Integration von ,Citizen Science'-Elementen; s. WiNo-Box 7 für die in WiNo vorgesehenen Instrumente).

Den Themenfeld-Teams wurden die untenstehenden Fragen gestellt, die sie in Form einer Projektskizze und ergänzt um einen Zeit- und Kostenplan einreichten. Wie in Frage 10 ersichtlich, wurden in WiNo spezifische Finanzmittel für die transdisziplinäre Zusammenarbeit bereitgestellt: Ein Wissenschaft-Praxis-Tandem-Programm sollte es Wissenschaftler(inne)n und Praxisakteuren ermöglichen, die Arbeit der jeweils, anderen Seite' kennenzulernen; Mobilitätsstipendien erlaubten es Wissenschaftler(inne)n und Akteuren aus der Region, Erfahrungen mit anderen Nationalparks im In- und Ausland auszutauschen; in forschenden Lehr- und Lernprojekten bearbeiteten Studierende Projektthemen direkt vor Ort. Die Fragen im Einzelnen:

\section{Problemstellung}

Wie ist die Ausgangslage, was ist der Forschungsanlass?

\section{Zielstellung des Projektes und Forschungsfrage(n)}

Welches Ziel verfolgt das Projekt; welche Forschungsfragen sollen beantwortet werden?

\section{Methoden/Methodologie und Datengrundlage}

Welche Methodik/Methodologie soll zum Einsatz kommen? Welche Datengrundlage liegt vor bzw. welche Daten müssen erhoben werden?

\section{Relevanz des Themas}

Wo sehen Sie die Relevanz des Themas für die nachhaltige Entwicklung der Region Nordschwarzwald bzw. für einzelne Akteure oder Akteursgruppen? Hat das Projekt das Potential zu der im Antrag als Projektziel angegebenen Nachhaltigkeitstransformation beizutragen?

\section{Abgrenzung von laufenden Forschungsarbeiten}

1) Welche ähnlich gelagerten Forschungsprojekte haben Sie bzw. die beteiligten Institutionen in den letzten Jahren dazu bereits bearbeitet - wie grenzt sich das beantragte Forschungsprojekt davon ab?

2) Welche Arbeiten zu diesem Thema werden bereits durch die Verwaltung des Nationalparks Schwarzwald durchgeführt? Welchen darüber hinausgehenden Mehrwert liefert Ihr Projekt?

\section{Vernetzung mit anderen Themenfeldern}

Welche Möglichkeit sehen Sie, Ihr Forschungsprojekt mit den Projekten der anderen Themenfelder zu verknüpfen? 


\section{Ergebnisse des Wissensdialogs}

Welche Anregungen, Kritik, Hinweise haben Sie im Verlauf des Wissensdialogs erhalten? Welcher Kritik sind Sie aus welchen Gründen gefolgt bzw. aus welchen Gründen nicht gefolgt; welche Anregungen und Hinweise haben Sie in Ihre Skizze integriert - und wie?

\section{Zukünftige Zusammenarbeit mit regionalen Akteuren und Bevölkerung}

Wie wollen Sie im Projektverlauf mit regionalen Akteuren bzw. der Bevölkerung zusammenarbeiten bzw. die Zusammenarbeit zwischen den Akteuren fördern?

\section{Einbindung studentischer Arbeiten}

Welche der Fragestellungen Ihres Projektes wollen Sie als studentische Arbeiten (z. B. Masterarbeiten) bearbeiten lassen? Ggf. wie?

\section{Einbindung der WiNo-Instrumente transdisziplinärer Zusammenarbeit}

Wie wollen Sie die unterschiedlichen Instrumente trans- und interdisziplinärer Zusammenarbeit (Wissenschaft-Praxis-Tandems, Mobilitätsstipendien, Lehrprojekte, Gender \& Diversity) in Ihrem Projekt nutzen bzw. anwenden?

\section{Zeitplanung}

\section{Budgetplanung}

Die von den Themenfeldgruppen aufgrund der Wissensmesse vorgenommenen Veränderungen reichten von Konkretisierungen bis hin zu thematischen Neuausrichtungen, wie die Titel der eingereichten Teilprojekte zeigen (vgl. Titel in WiNo-Box 3):

1. Tourismus und Mobilität (s. Bachinger et al. 2018)

2. Heute Kulturwald - morgen Urwald? Restaurationsmaßnahmen als Impuls für eine natürliche Waldentwicklung

3. Untersuchungen zur Ausbreitungsdynamik des Buchdruckers (Ips typographus) im Nationalpark Schwarzwald

4. Landnutzung und Waldentwicklung im Nordschwarzwald - gestern, heute, morgen

5. Entwicklung eines GIS-gestützten Planungsinstruments für die Wegeplanung im Nationalpark und in der Region Nordschwarzwald

6. Wahrnehmung und Bewertung von Wildtieren und deren Management in der Region Nordschwarzwald

7. Lokales Wissen zum Nationalpark Schwarzwald

WiNo-Box 7: Der WiNo-Fragenkatalog für die Ausarbeitung der Projektskizzen 
Schritt 13: Kommunikation des beschlossenen Forschungsprogramms, Dokumentation und Abschluss der Programmentwicklung

Das Kernteam kommuniziert das beschlossene Forschungsprogramm und die damit verbundene Entscheidung über die Finanzierung der Teilprojekte an die beteiligten Wissenschafts-Praxis-Teams. Das Konsortium des Gesamtprojekts besteht aus dem Kernteam und den geförderten Wissenschafts-Praxis-Teams. Wichtig erscheint für die zukünftige Zusammenarbeit im Konsortium, dass bei der Kommunikation der Entscheidung alle Beiträge der Wissenschafts-Praxis-Teams, insbesondere wenn sie in unterschiedlichem Maße mit Finanzmitteln bedacht werden, gleichermaßen anerkennend gewürdigt werden. Denn die Erarbeitung von Ideen für Teilprojekte, deren öffentliche Präsentation und Weiterentwicklung zu Projektskizzen sind mit einem hohen Aufwand verbunden, und dieser Aufwand muss ideell honoriert werden. Die Wissenschafts-Praxis-Teams werden aufgefordert, sich in Form einer kurzen schriftlichen Stellungnahme (ca. eine Seite) zu den Empfehlungen des Projektbeirates zu äußern. Hier ist z. B. auch denkbar, dass ein Teilprojekt begründet, warum es einer Empfehlung nicht folgen kann, bzw. ausführt, wie es eine Empfehlung umsetzen möchte.

Anschließend wird das Forschungsprogramm den an der Wissensmesse involvierten Akteuren und einer breiteren Öffentlichkeit vorgestellt, und es wird um die weitere Beteiligung an den Forschungsaktivitäten des Projekts geworben. Abhängig vom Projektkontext und den zur Verfügung stehenden Ressourcen kann dies im Rahmen einer Abendveranstaltung, eines Newsletters und/oder per Pressemitteilung erfolgen. Dabei gebührt auch den einzig an der Wissensmesse beteiligten Akteuren Dank. Das Kernteam sorgt abschließend für die Dokumentation des Entscheidungsprozesses, um auch zukünftig transparent Auskunft über Art und Ergebnis der Entscheidung geben zu können.

\section{Die beteiligten Akteure und ihre Rollen im Überblick}

In Tab. 2 sind die auf dem Weg zu einem transdisziplinären Forschungsprogramm zentralen Akteure überblicksartig zusammengestellt. Für die vier ausgewiesenen Gruppen zeigen sich spezifische Beteiligungs- und Entscheidungsprofile:

Praxisakteure, die nicht direkt an der Entwicklung von Teilprojekt-Ideen beteiligt sind, spielen eine wichtige Rolle, sie übernehmen im beschriebenen Vorgehen jedoch keine Initiativfunktion, sondern werden weitgehend , auf Einladung' eingebunden. Sie fungieren - in verschiedenen Phasen des Prozesses - als Ideengeber. Eine formale Entscheidungsbefugnis kommt ihnen nicht zu. 
Tabelle 2: Modus der Beteiligung und Entscheidungsfindung.

\begin{tabular}{|c|c|c|}
\hline Akteure & Modus der Beteiligung & Modus der Entscheidung \\
\hline $\begin{array}{l}\text { Fachöffentlich- } \\
\text { keit generell, } \\
\text { organisierte } \\
\text { Öffentlichkeit } \\
\text { generell, breite } \\
\text { Öffentlichkeit }\end{array}$ & $\begin{array}{ll}\text { - } & \text { als Interviewpartner(innen) im } \\
\text { Rahmen der einleitenden Be- } \\
\text { fragung (Schritt 2) } \\
\text { - } \quad \text { als Ideengeber(innen) und } \\
\text { Diskutant(inn)en im Rahmen } \\
\text { der Wissensmesse (Schritt 10) }\end{array}$ & $\begin{array}{l}\text { - ohne Entscheidungsbe- } \\
\text { fugnis } \\
\text { - Einfluss über Wissens- } \\
\text { und Themen-Inputs }\end{array}$ \\
\hline $\begin{array}{l}\text { Wissenschafts- } \\
\text { Praxis-Teams } \\
\text { (bestehend aus } \\
\text { Wissenschaftle- } \\
\text { r(inne)n, Praxis- } \\
\text { akteuren aus } \\
\text { Fachöffentlich- } \\
\text { keit und organi- } \\
\text { sierter Öffent- } \\
\text { lichkeit) }\end{array}$ & $\begin{array}{l}\text { - } \text { fachlicher und methodischer } \\
\text { Beitrag bei Erarbeitung von } \\
\text { Vorschlägen für Teilprojekte } \\
\text { (Schritt 7) } \\
\text { - } \quad \text { inhaltliche Mitwirkung an } \\
\text { Vorbereitung und Durchfüh- } \\
\text { rung der Wissensmesse } \\
\text { (Schritte 9, 10) } \\
\text { - } \\
\text { Ausarbeitung von Projektskiz- } \\
\text { zen (Schritt 11) }\end{array}$ & 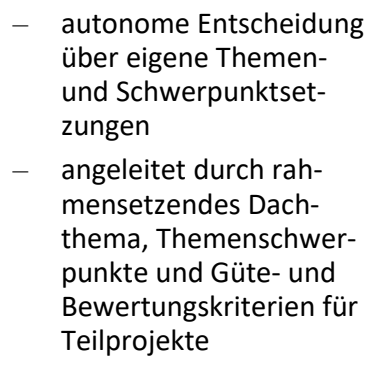 \\
\hline $\begin{array}{l}\text { Kernteam } \\
\text { (bestehend aus } \\
\text { Wissenschaft- } \\
\text { ler(inne)n, Pra- } \\
\text { xisakteuren aus } \\
\text { Fachöffentlich- } \\
\text { keit und organi- } \\
\text { sierter Öffent- } \\
\text { lichkeit) }\end{array}$ & $\begin{array}{l}\text { Zentraler ,Motor' des Gesamtpro- } \\
\text { jekts (als prozessgestaltende und - } \\
\text { steuernde Instanz): } \\
\text { - } \quad \text { Organisation und Durchfüh- } \\
\text { rung der einleitenden Befra- } \\
\text { gung (Schritt 2) } \\
-\quad \text { Festlegung der Themen- } \\
\text { schwerpunkte (Schritt 3) } \\
-\quad \text { Einsetzung des Projektbeirats } \\
\text { (Schritt 4) } \\
-\quad \text { Erarbeitung von Auswahlkrite- } \\
\text { rien für Teilprojekte (Schritt 5) } \\
-\quad \text { Ausschreibung von Themen- } \\
\text { schwerpunkten (Schritt 6) } \\
-\quad \text { Vorbereitung und Durchfüh- } \\
\text { rung der Wissensmesse } \\
\text { (Schritte } 9 \text { und 10) } \\
-\quad \text { Festlegung des Forschungs- } \\
\text { programms (Schritt 12) } \\
\text { Kommunikation des For- } \\
\text { schungsprogramms und Do- } \\
\text { kumentation der Entschei- } \\
\text { dungsfindung (Schritt 13) }\end{array}$ & 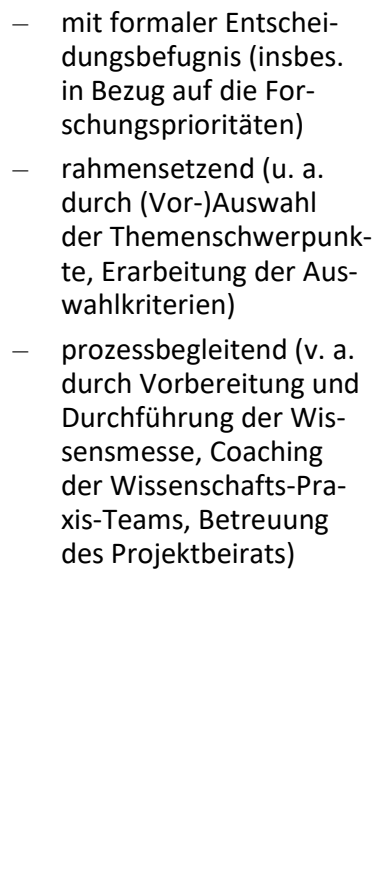 \\
\hline
\end{tabular}




\begin{tabular}{|c|c|c|}
\hline Akteure & Modus der Beteiligung & Modus der Entscheidung \\
\hline $\begin{array}{l}\text { Projektbeirat } \\
\text { (bestehend aus } \\
\text { Wissenschaft- } \\
\text { ler(inne)n, Pra- } \\
\text { xisakteuren aus } \\
\text { Fachöffentlich- } \\
\text { keit und organi- } \\
\text { sierter Öffent- } \\
\text { lichkeit) }\end{array}$ & 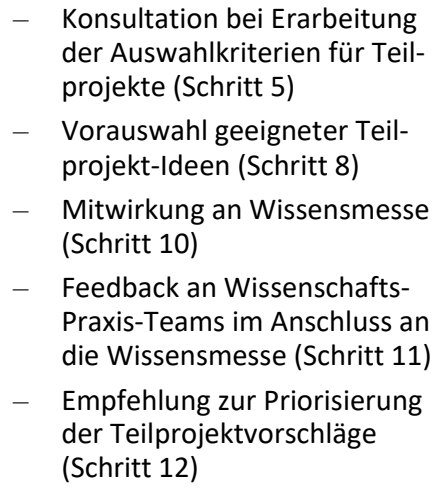 & $\begin{array}{l}\text { - } \begin{array}{l}\text { ohne formale Entschei- } \\
\text { dungsbefugnis }\end{array} \\
-\quad \text { Einfluss aufgrund seiner } \\
\text { Beratungsfunktion }\end{array}$ \\
\hline
\end{tabular}

Deutlich aktiver ist das Profil jener Akteure, die den Wissenschafts-Praxis-Teams angehören; dazu gehören - im Sinne des transdisziplinären Anspruchs der Teilprojekte - neben Wissenschaftler(inne)n auch Praxisakteure aus Fachöffentlichkeit und organisierter Öffentlichkeit. Diese sind für ihre Bereiche, Themensetzer', jedoch sind sie in ihrer Themensetzung nicht völlig frei, sondern über das vom Kernteam definierte Dachthema, die Themenschwerpunkte und die Güteund Bewertungskriterien für die Teilprojekte sowohl inhaltlich als auch methodisch gebunden.

Die aktivste und entscheidungsmächtigste Rolle spielt im beschriebenen Vorgehen das Kernteam, dem sowohl Wissenschaftler(innen) als auch Praxisakteure (aus Fachöffentlichkeit und organisierter Öffentlichkeit) angehören. Das Kernteam ist der zentrale ,Motor' des Gesamtprojekts. Ihm fallen inhaltlich und methodisch rahmensetzende wie auch operativ prozessbegleitende Aufgaben zu. Darüber hinaus liegen beim Kernteam zahlreiche formale Entscheidungskompetenzen, wie insbesondere die Entscheidung über die Priorisierung der Teilprojekte und die damit verbundenen Budgetzuweisungen.

Als letzter wichtiger Akteur in unserem Vorgehen ist schließlich der Projektbeirat zu nennen, dem ebenfalls sowohl Wissenschaftler(innen) als auch Praxisakteure (aus Fachöffentlichkeit und organisierter Öffentlichkeit) angehören. Der Projektbeirat ist über die verschiedenen Verfahrensschritte hinweg eingebunden, jedoch stets in einer ausschließlich beratenden Funktion, d. h. ohne formale Entscheidungsbefugnis. 


\section{Die Herausforderungen: Vom Umgang mit zentralen Spannungslinien des Verfahrens}

Während in Kapitel 2 ein konkretes Vorgehen zum Erarbeiten von Forschungsthemen und -fragen im Austausch zwischen Wissenschaft und Praxis entworfen und begründet wurde, wollen wir im abschließenden Kapitel eine etwas distanziertere Reflexion zu den in den oben beschriebenen Prozessschritten zum Teil inhärent innewohnenden Spannungen anstellen. Diese Reflexion stützt sich auf Erfahrungen, wie wir sie im Reallabor-Projekt WiNo über gut drei Jahre hinweg gemacht haben. Methodisch greifen wir dabei auf eine qualitative Befragung zurück, welche in WiNo etwa zur Hälfte der Projektlaufzeit durchgeführt wurde (ausführlicher dazu s. Rhodius et al. 2016). Dabei wurden 13 von insgesamt rund 20 WiNo-Wissenschaftler(innen) zu ihren Erfahrungen mit dem transdisziplinären Design interviewt. Die ausgewählten Interviewpartner(innen) repräsentierten dabei das inhaltliche Spektrum und die verschiedenen Gremien des Projektes. Die Ergebnisse dieser Befragung werden hier mit persönlichen Einsichten, welche die Autorin und der Autor in begleitender Beobachtung in verschiedenen Rollen im Projekt sammeln konnten, trianguliert (zu weiteren Ergebnissen s. Rhodius et al. 2016; Pregernig et al. 2017).

Aufbauend auf unseren Erfahrungen in WiNo wollen wir unsere abschließenden Reflexionen entlang von drei zentralen Spannungslinien herausarbeiten: Diese beziehen sich auf (1) die komplexe Schrittfolge, die dem oben beschriebenen Vorgehen innewohnt; (2) die Entscheidung, explizite Anreize für Forscher(innen) zu schaffen, sich auf transdisziplinäre Prinzipien und Formate einzulassen; und (3) das Bestreben, sehr unterschiedliche Gruppen von Praxisakteuren in die Entwicklung des Forschungsprogramms einzubeziehen.

\section{1) Herausforderungen des sequentiellen Vorgehens}

Dem in Kapitel 2 vorgestellten Vorgehen liegt ein zeitlich ausgedehntes und stark sequentielles Prozessformat zugrunde. Im Zentrum steht die eintägige Wissensmesse, welche jedoch flankiert wird von einer sich über mehrere Monate erstreckenden Phase der Vorbereitung und einer sich zumindest über mehrere Wochen erstreckenden Phase der Nachbereitung.

In WiNo erwies sich das sequentielle Vorgehen als herausfordernd, jedoch grundsätzlich als erfolgreich: In der oben erwähnten Befragung der WiNo-Forscher(innen) zeigten sich die meisten mit dem gewählten Ansatz der Forschungsprogrammformulierung im Austausch zwischen Wissenschaft und Praxis grundsätzlich zufrieden. Die Befragten erkannten an, dass das gewählte Format die Forscher(innen) zwang, „regionalen Akteuren mal zuhören zu müssen“, was in Folge zur „Öffnung von Forschungs-Agenden“ und zum „Experimentieren mit 
neuen Ideen" führte. Gleichzeitig gab es in WiNo aber auch Stimmen, welche die rund neun Monate, die zwischen Projektstart und finaler Festlegung des Forschungsprogramms vergingen, als ,Zeitverlust für die eigentliche Forschungsarbeit" wahrnahmen.

Auch wenn das oben beschriebene Vorgehen in manchen Punkten von dem in WiNo realisierten Ansatz abweicht, so lassen sich unserer Einschätzung nach doch verallgemeinerbare Schlussfolgerungen ziehen. So steckt hinter den scheinbar widersprüchlichen Stimmen wohl ein inhärentes Spannungsverhältnis zwischen dem Wunsch von Forscher(inne)n nach Vorhersehbarkeit und Planbarkeit von Forschungsprozessen auf der einen Seite und der notwendigen Flexibilität von Prozeduren im Kontext eines Reallabors oder transdisziplinärer und transformativer Forschung allgemein auf der anderen Seite. Im oben beschriebenen Vorgehen wurde versucht, einigen dieser Spannungen aktiv entgegenzuwirken, etwa durch frühe Rollenklärungen und ein klares Erwartungsmanagement oder durch die Festlegung verbindlicher Entscheidungskriterien und transparenter Entscheidungsregeln. Demgegenüber gibt es jedoch Spannungen, die nicht so einfach aufgelöst werden können: So braucht es für die Umsetzung einer so ambitionierten Schrittfolge einfach ausreichend Zeit, insbesondere auch, um Lernprozesse zwischen den verschiedenen Akteuren zu ermöglichen. ${ }^{2}$ Dadurch, dass in WiNo das gesamte Forschungsteam von Beginn an in den Prozess involviert war, wurden durch das langwierige, gestufte Vorgehen die Kapazitäten mancher Forscher(innen) wohl überbelastet. Im hier beschriebenen Vorgehen wurde dem dahingehend Rechnung getragen, dass die ersten Schritte nur von einem kleinen Kernteam durchlaufen werden und dass erst später ein breiterer Kreis von Forscher(inne)n in das Projekt hereingeholt wird.

\section{2) Herausforderungen der Steuerung von Transdisziplinarität über Anreize}

Neben dem schrittweisen Vorgehen zeichnet sich unser oben vorgestelltes Vorgehen auch dadurch aus, dass es explizite Anreize für Transdisziplinarität schafft. In der einschlägigen Literatur werden Anreizmodelle kaum vorgeschlagen und diskutiert; Regeln „guten transdisziplinären Arbeitens“ sollen demgegenüber vor allem über Qualitätskriterien (z. B. Bergmann et al. 2005) und Managementleitfäden (z. B. Defila et al. 2006) sichergestellt werden.

Um ein möglichst konsequentes transdisziplinäres Forschungsdesign umsetzen zu können, entschied man sich in WiNo dafür, explizite, formale Anreizstrukturen zu schaffen: Forscher(innen) mussten ihre Projektideen einem kompetitiven Auswahlprozess unterziehen, und am Ende entschied ein Steuerungsgremium, welche

2 Eine Analyse der spezifischen WiNo-Lernprozesse findet sich in Bachinger und Rhodius (2018). 
Teilprojekte gefördert und mit welchen Budgets diese ausgestattet werden würden. Wie die oben erwähnte Befragung zeigte, empfanden einige der Forsche$\mathrm{r}$ (innen) das kompetitive Element als hilfreich, insbesondere, um mit den regionalen Akteuren in einen Dialog , auf Augenhöhe' zu treten. Ein anderer Teil der Befragten monierte hingegen den mit dem Wettbewerbselement verbundenen Mangel an Planungssicherheit. Vergleicht man jedoch die Projektideen, wie sie zu Beginn des WiNo-Prozesses von den Forschungsteams eingereicht wurden, mit jenen, wie sie am Ende des Auswahlprozesses vorlagen, so zeigt sich bei der Mehrzahl der Teilprojekte eine eindeutige Stärkung der Berücksichtigung der Perspektiven von Praxisakteuren. Dies kann als Indikator für die Effektivität der im wettbewerblichen Verfahren angebotenen Anreize gesehen werden.

\section{3) Herausforderungen in der Zusammenarbeit mit unterschiedlichen Akteursgruppen}

Eine dritte Herausforderung, die in allgemeiner Hinsicht vielen transdisziplinären Formaten gemein ist, die aber bei der transdisziplinären Entwicklung eines Forschungsprogramms spezifische Merkmale aufzeigt, ist jene der Einbindung von Praxisakteuren. Mit ihrem programmatischen Bekenntnis zu gesellschaftlicher Transformation legt die Reallabor-Community großes Augenmerk auf die Einbindung zivilgesellschaftlicher Akteure (oft mit einer Betonung der breiten Bevölkerung), und sie sieht diese als wichtige Change Agents in Richtung Nachhaltigkeit; ihnen soll in Reallabor-Projekten dementsprechend eine aktive Rolle zuerkannt werden (s. Grießhammer und Brohmann 2015, Parodi et al. 2016).

Der breite Konsens in Bezug auf Akteurseinbindung lässt jedoch häufig übersehen, dass damit oft auch ein Zielkonflikt (i. S. eines Trade offs) einhergeht: Reallabor-Forschung versucht oftmals, sowohl eine breite Öffentlichkeit einzubinden als auch spezifische Fachöffentlichkeiten. Am Beispiel von WiNo lässt sich das damit verbundene Spannungsverhältnis, dem wohl auch das oben beschriebene Vorgehen nur schwer entkommen kann, gut aufzeigen: In WiNo wurde versucht, drei Typen von außerwissenschaftlichen Akteursgruppen einzubinden: Die aktivste Rolle wurde der Nationalparkverwaltung zugesprochen, zu welcher das Leitungsteam wie auch einzelne Teilprojekte regelmäßigen - zumeist bilateralen Kontakt pflegten. Daneben gab es eine Reihe von organisierten regionalen Gruppen, die aktiv z. B. über Workshops oder gemeinsam organisierte Veranstaltungen in die Teilprojekte eingebunden waren. Demgegenüber wurde eine breite Öffentlichkeit in WiNo nur sporadisch eingebunden. Selbst über das zentrale Instrument des Wissenschaft-Praxis-Austauschs, die eintägige Wissensmesse, vermochte man nicht, ein breites Spektrum an Bürger(inne)n anzusprechen: Etwa 80 \% der teilnehmenden Praxisakteure waren Repräsentant(inn)en aus Wirtschaft, Verbänden, Politik und Verwaltung; nur rund $20 \%$ waren ,normale` Bürger(innen). Diese Selektivität der Teilnahme ist sowohl aus der Literatur zu partizipati- 
ven Verfahren allgemein (Alcántara et al. 2016) wie auch speziell zu transdisziplinären Prozessen (Lang et al. 2012) bekannt.

In der Befragung der WiNo-Forscher(innen) wurde dieser Sachverhalt interessanterweise sehr unterschiedlich bewertet: Ein Teil der Befragten war über die schwache Beteiligung von Bürger(inne)n sehr enttäuscht; für sie hatte WiNo sein „Beteiligungsziel eindeutig verfehlt“. Im Gegensatz dazu waren andere Wissenschaftler(innen) der Meinung, dass man damit „genau die richtigen Leute“ adressiert hätte, weil im Kontext der Formulierung eines Forschungsprogramms nur die Einbindung professioneller, einschlägig sachkundiger Akteure zu ,ergiebigen Diskussionen und nützlichen Inputs“ führen würde. Demnach wären ,normale“ Bürger(innen) mit der Aufgabe, Forschungsfragen zu formulieren, ohnehin überfordert. Für uns scheint das beschriebene Spannungsverhältnis keineswegs WiNospezifisch, sondern weitgehend für andere Typen der transdisziplinären Forschung verallgemeinerbar zu sein (für einen Expertise-Zugang sprechen sich z. B. auch Di Giulio et al. 2016 aus). Basierend auf den WiNo-Erfahrungen konzentriert sich das hier beschriebene Vorgehen daher nun auf die Einbeziehung von Fachöffentlichkeit und organisierter Öffentlichkeit.

Wie eingangs dargestellt, ist das beschriebene Vorgehen klar forschungsorientiert. Im Zentrum stehen nicht Maßnahmen der Einbindung von Bürger(inne)n in einzelne Realexperimente oder ähnliche praxisorientierte Prozesse. Als Ergebnis steht am Ende vielmehr ein kohärentes Forschungsprogramm, das die Forschung im Reallabor oder einem ähnlichen transdisziplinären Format für einen längeren, zumindest ein bis zwei Jahre umfassenden Zeitraum strukturieren und anleiten soll. Dementsprechend zeit- und ressourcenaufwendig ist auch das vorgestellte Vorgehen; es verlangt sowohl den beteiligten Wissenschaftler(inne)n als auch den eingebundenen Praxisakteuren ein hohes $\mathrm{Ma}$ an Einsatz und Engagement ab. Eine fast notwendige Konsequenz daraus ist, dass in diesem Modell einer breiteren Öffentlichkeit - wie oben beschrieben - nur eine untergeordnete Rolle zukommen kann. Um einer daraus möglicherweise resultierenden expertokratischen Verengung entgegenzuwirken und das Projekt besser und breiter im Raum des Projekts zu verankern, empfiehlt es sich, begleitend zum oder anknüpfend an das hier beschriebene Verfahren Maßnahmen zum Dialog mit der breiten Öffentlichkeit durchzuführen. In WiNo wurde dazu die Reihe „Wissensdialog vor Ort“ ins Leben gerufen: Hier luden die Teilprojekte mit Exkursionen, Vorträgen und Aktionen im Feld zum Gespräch zwischen Wissenschaft, Fachöffentlichkeit, organisierter Öffentlichkeit und breiter Öffentlichkeit ein. Die Reihe fand große Resonanz, und wurde der Wissensdialog noch überwiegend von Praxisakteuren aus der Fachöffentlichkeit und der organisierten Öffentlichkeit besucht, so nahmen bei den regionalen Veranstaltungen auch Bürger(innen) teil. Die Ergebnisse der Gespräche dieser Veranstaltungsreihe beeinflussten wiederum die Arbeit der Teilprojekte, z. B. indem neue Fragestellungen aufgeworfen wurden, sich Anwen- 
dungsmöglichkeiten für Forschungsergebnisse auftaten oder Ideen für zukünftige Projekte benannt wurden. Das hier vorgeschlagene, klar auf Wissenschaft und Praxis (verstanden als Fachöffentlichkeit und organisierte Öffentlichkeit) fokussierte Verfahren zur Definition von Forschungsfragen und -themen in transdisziplinären Projekten lässt sich also gut mit Maßnahmen zur Einbeziehung der breiteren Öffentlichkeit kombinieren.

\section{Dank}

Die Autorin und der Autor danken Sophia Alcántara, Marius Gantert, Anja-Lisa Hirscher, Felix M. Piontek, Friedemann Schwenkreis und Andreas Seebacher für die Diskussionen über frühere Fassungen des Textes. Insbesondere möchten sie Sarah Meyer und Stefan Zimmermann sowie zwei anonymen Gutachter(inne)n für die konkreten Hinweise zur Verbesserung des Textes im Rahmen des internen und externen Reviews danken. Schließlich danken die Autorin und der Autor den beiden Herausgebenden, Rico Defila und Antonietta Di Giulio, für ihre Rückmeldungen zum Text.

\section{Literatur}

Alcántara, S., Bach, N., Kuhn, R., \& Ullrich, P. (2016). Demokratietheorie und Partizipationspraxis: Analyse und Anwendungspotentiale deliberativer Verfahren. Wiesbaden: Springer VS.

Arbter, K. (2012). Praxisbuch Partizipation: Gemeinsam die Stadt entwickeln. Wien: Magistrat der Stadt Wien.

Arnold, A., \& Piontek, F. M. (2018). Zentrale Begriffe im Kontext der Reallaborforschung. In R. Defila \& A. Di Giulio (Hrsg.), Transdisziplinär und transformativ forschen. Eine Methodensammlung (S. 143-154). Wiesbaden: Springer VS.

Beecroft, R., Trenks, H., Rhodius, R., Benighaus, C., \& Parodi, O. (2018).

Bachinger, M., Bleher, D., Rau, H., \& Prieß, R. (2018). Die „WiNo-Methode“ zur Identifikation von Wissen in transdisziplinären Netzwerken mithilfe der Wissensbilanzierung. In R. Defila \& A. Di Giulio (Hrsg.), Transdisziplinär und transformativ forschen. Eine Methodensammlung (S. 301-327). Wiesbaden: Springer VS.

Bachinger, M., \& Rhodius, R. (2018). Anforderungen an die Governance von transdisziplinären Lernprozessen in Reallaboren. Das Beispiel des „Wissensdialogs Nordschwarzwald (WiNo)“. Berichte. Geographie und Landeskunde, 91 (1), (S. 81-96). (im Druck).

Bergmann, M., Brohmann, B., Hoffmann, E., Loibl, M. C., Rehaag, R., Schramm, E., \& Voß, J. P. (2005). Qualitätskriterien transdisziplinärer Forschung: Ein Leitfaden für die formative Evaluation von Forschungsprojekten. Frankfurt a. M.: ISOE. 
Blättel-Mink, B., Kastenholz, H. G., Schneider, M., \& Spurk, A. (2003). Nachhaltigkeit und Transdisziplinarität: Ideal und Forschungspraxis. Stuttgart: Akademie für Technikfolgenabschätzung in Baden-Württemberg.

Defila, R., \& Di Giulio, A. (2018a). Partizipative Wissenserzeugung und Wissenschaftlichkeit - ein methodologischer Beitrag. In R. Defila \& A. Di Giulio (Hrsg.), Transdisziplinär und transformativ forschen. Eine Methodensammlung (S. 39-67). Wiesbaden: Springer VS.

Defila, R., \& Di Giulio, A. (2018b). Reallabore als Quelle für die Methodik transdisziplinären und transformativen Forschens - eine Einführung. In R. Defila \& A. Di Giulio (Hrsg.), Transdisziplinär und transformativ forschen. Eine Methodensammlung (S. 935). Wiesbaden: Springer VS.

Defila, R., Di Giulio, A., \& Scheuermann, M. (2006). Forschungsverbundmanagement: Handbuch für die Gestaltung inter- und transdiziplinärer Projekte. Zürich: vdf Hochschulverlag.

Di Giulio, A., Defila, R., \& Brückmann, Th (2016). „Das ist halt das eine ... Praxis, das andere ist Theorie" - Prinzipien transdisziplinärer Zusammenarbeit im Forschungsalltag. In R. Defila \& A. Di Giulio (Hrsg.), Transdisziplinär forschen - zwischen Ideal und gelebter Praxis. Hotspots, Geschichten, Wirkungen (S. 189-286). Frankfurt a. M., New York: Campus.

Grießhammer, R., \& Brohmann, B. (2015). Wie Transformationen und gesellschaftliche Innovationen gelingen können. Dessau-Roßlau: Umweltbundesamt.

Hoyningen-Huene, P. (1987). Context of Discovery and Context of Justification. Studies In History and Philosophy of Science, 18 (4), (S. 501-515).

Königswieser, R., \& Keil, M. (Hrsg.). (2000). Das Feuer großer Gruppen: Konzepte, Designs, Praxisbeispiele für Großveranstaltungen. Stuttgart: Klett Cotta.

Lang, D. J., Wiek, A., Bergmann, M., Stauffacher, M., Martens, P., Moll, P., . . Thomas, C. J. (2012). Transdisciplinary research in sustainability science: practice, principles, and challenges. Sustainability Science, 7 (1), (S. 25-43).

Moser, S. C. (2016). Can science on transformation transform science? Lessons from codesign. Current Opinion in Environmental Sustainability, 20, (S. 106-115).

Parodi, O., Beecroft, R., Albiez, M., Quint, A., Seebacher, A., Tamm, K., \& Waitz, C. (2016). Von „Aktionsforschung“ bis „Zielkonflikte“ - Schlüsselbegriffe der Reallaborforschung. Technikfolgenabschätzung - Theorie und Praxis, 25 (3), (S. 9-18).

Pregernig, M., Rhodius, R., \& Winkel, G. (2018). Design junctions in real-world laboratories: Analyzing experiences gained from the project „Knowledge Dialogue Northern Black Forest“". GAIA, 27 (S1), (S. 32-38). (im Druck).

Rhodius, R., Pregernig, M., \& Koch, B. (2016). Herausforderungen transdisziplinären Arbeitens im Reallabor,Wissensdialog Nordschwarzwald“. Technikfolgenabschätzung - Theorie und Praxis, 25 (3), (S. 19-25). 
Seliger, R. (2008). Einführung in Großgruppen-Methoden. Heidelberg: Carl Auer Compact.

Weingart, P. (1993). Close Encounters of the Third Kind: Science and the Context of Relevance. In T. Brante, S. Fuller, \& W. Lynch (Hrsg.), Controversial Science: From Content to Contention (S. 153-174). Albany: SUNY Press.

Wiek, A. (2007). Challenges of Transdisciplinary Research as Interactive Knowledge Generation Experiences from Transdisciplinary Case Study Research. GAIA, 16 (1), (S. 52-57).

Will, H., Wünsch, U., \& Polewsky, S. (2009). Info-, Lern- und Change-Events: Ideenbuch für Veranstaltungen. Weinheim: Beltz.

Open Access Dieses Kapitel wird unter der Creative Commons Namensnennung 4.0 International Lizenz (http://creativecommons.org/licenses/by/4.0/deed.de) veröffentlicht, welche die Nutzung, Vervielfältigung, Bearbeitung, Verbreitung und Wiedergabe in jeglichem Medium und Format erlaubt, sofern Sie den/die ursprünglichen Autor(en) und die Quelle ordnungsgemäß nennen, einen Link zur Creative Commons Lizenz beifügen und angeben, ob Änderungen vorgenommen wurden.

Die in diesem Kapitel enthaltenen Bilder und sonstiges Drittmaterial unterliegen ebenfalls der genannten Creative Commons Lizenz, sofern sich aus der Abbildungslegende nichts anderes ergibt. Sofern das betreffende Material nicht unter der genannten Creative Commons Lizenz steht und die betreffende Handlung nicht nach gesetzlichen Vorschriften erlaubt ist, ist für die oben aufgeführten Weiterverwendungen des Materials die Einwilligung des jeweiligen Rechteinhabers einzuholen. 